\title{
Research on synthesis and self-healing properties of interpenetrating network hydrogels based on reversible covalent and reversible non-covalent bonds
}

\author{
Xiaowen Huang ${ }^{1} \cdot$ Xiaofei Wang $^{1} \cdot$ Chuanying Shi $^{1} \cdot$ Yang Liu $^{1} \cdot$ Yanyan Wei ${ }^{1}$
}

Received: 4 August 2019 / Accepted: 8 May 2020 / Published online: 5 January 2021

(C) The Author(s) 2021

\begin{abstract}
First of all, we will provide a brief background on the self-healing hydrogels we produced which are suitable for the complex environment of nature. In this paper, disulfide bonds and acylhydrazone bonds can be combined in SH-WPU and hydrogen bonds existed in PAMAM. And the hydrogel can achieve self-healing under acid, alkaline, neutral or light environment.Self-healing for $1 \mathrm{~h}$, $24 \mathrm{~h}$ and $48 \mathrm{~h}$, the self-healing efficiency is $31.58 \%, 49.84 \%$ and $87.35 \%$ respectively. This effect achieved the desired effect and the repair effect is more obvious than previous research results. The hydrogels have potential applications in the field of biomaterials.
\end{abstract}

Keywords Reversible covalent bond $\cdot$ Reversible non-covalent bond $\cdot$ Self-healing $\cdot$ IPN $\cdot$ Hydrogel

\section{Introduction}

Polymer materials have the characteristics of light weight, high strength, good corrosion resistance and excellent electrical insulation properties, so they are used in various fields. However, they are easily caused the loss of surface and internal micro-cracks [1, 2]. In the middle of the 1980s, the United States military put forward the concept of intelligent composite material with self-diagnosis and self-repair function, which inspired by the self-healing of the bodies. People defined the concept of the self-healing materials, which had the capacity of the self-judgment, self-control and self-repairing [3].

Self-healing materials are usually divided into capsulebased material, hollow fiber and intrinsic self-healing materials. The types of intrinsic self-healing include reversible covalent bonds (Diels-Alder bond, acylhydrazone bond, disulfide bond and three thioester bond etc.) and reversible noncovalent bonds (hydrogen bond, host guest interaction, hydrophobic interaction, and stacking interaction etc.). The first

Yanyan Wei

yywei@qust.edu.cn

1 Key Laboratory of Rubber-plastics, Ministry of Education/Shandong Provincial Key Laboratory of Rubber-plastics, School of Polymer Science and Engineering, Qingdao University of Science \& Technology, Qingdao, Shandong 266042, People's Republic of China capsule-based self-healing system was studied by White et al. [4-6] which based on the ring-opening metathesis polymerization of dicyclopentadiene. When the material was damaged, the fiber was broken by the cracks and the repair agent was flowed out to heal the cracks, so the mechanical properties and performance can recover to a certain degree. But these self-healing systems have some disadvantages, for intance the limited repair times and the new gaps were created by the release of repair agent in the microcapsules or hollow fibers, which became new defects. Therefore, scientists turned their attention to the intrinsic self-healing system [7].

Liu and Hsieh et al. [8] studied the synthesis of blend polymers of multifunctional maleimide monomers and furan monomers. After heating, materials could be healed by open-loop and closed-loop thermo-reversible reaction and can repeate several times. Besides, furan-terminated polyurethane pre-polymer and bismaleimide were used to synthesize thermo-reversible self-healing polyurethane based on DielsAlder reaction by $\mathrm{Yu}$ Zhengyang et al. [9]. The dynamic reversibility of acylhydrazone bonds were used to prepare a $\mathrm{pH}-$ responsive polymer gel by Deng et al. [10], which can achieve the phase transition of sol-gel under acidic conditions. Also, a new type of dynamic gel combined the acylhydrazone bonds and disulfide bonds was also prepared by Deng et al. [11]. The gel can be healed by reversible exchange of acylhydrazone bonds under acidic $(\mathrm{pH}=3.6)$ conditions or by reversible exchange of disulfide bonds under alkaline $(\mathrm{pH}=9)$ conditions. Also, in the neutral $(\mathrm{pH}=7)$ conditions, the materials could be 
self-healed by the acylhydrazone bonds, which were helped by catalyst aniline. However, more time was required by the reversible covalent bond self-healing systems, so the reversible non-covalent bonds self - healing systems [12] were developed by many scientists. A super-molecular elastomer contained amide ethyl and healed by hydrogen bonds was synthesized by Cordier et al. [13] which could restore its original strength. Besides, the host-guest interaction of dibenzo24-crown- 8 and secondary ammonium salts were used to prepare the self-healing super-molecular gels by Zhang et al. [14, 15]

Recently, some scientists have focused their attention on the research of interpenetrating networks (IPN). Because there are no strong chemical bonds between the various components of the hydrogels network, and the relative independence of the respective properties can be maintained, but their mechanical strength and flexibility can be improved [16-18]. IPN structure included full-IPN [19] and semi-IPN [20] and preparation methods included one step method [21,22] and two steps method [23, 24].

In this paper, full-IPN is prepared by two steps method, Polyurethane (SH-WPU) is synthesized as the first network. And the polyamide amine (PAMAM) is obtained as the second network. We got this hydrogel with high mechanical strength and rapid self-healing ability for the first time. The hydrogels self-repair for $48 \mathrm{~h}$, and the repair efficiency is as high as $87.35 \%$ which is more obvious than previous research results.

\section{Experimental section}

\section{Materials}

Dimethyl 3,3'-dithiobispropionate which accounts for $96 \%$ in $100 \mathrm{~g}$ and comes from Shanghai Aladdin Biochemical Technology Limited Company.

Methanol,Hydrazine hydrate $80 \%$,Isopropanol, glacial acetic acid, absolute ethanol, Dimethyl sulfoxide called DMSO, Dibutyltin dilaurate called DBTDL and N, N'-methylene bisacrylamide called MBA which are AR.

4-hydroxybenzaldehyde which is analytical reagent comes from Tianjin Guangfu Fine Chemical Industry Research Institute.

Molecular sieve (1000 meshes) is used to remove its water.

Isophorone diisocyanate called IPDI, which constitutes 99.5\% in $100 \mathrm{~g}$ and comes from Bayer of Germany.

Polyethylene glycol 1000 called PEG-1000, which is experimental reagent and comes from Tianjin Damao Chemical Reagent Factory. Also, its water is removed at $100^{\circ} \mathrm{C}$ under reduced pressure environment.

Hexamethylene diisocyanate trimer called HDI trimer, which isocyanate groups account for $19.6 \%$ in $100 \mathrm{~g}$ and come from Bayer of Germany.
$\mathrm{N}$-aminoethylpiperazine called AEPZ, which accounts for 99.5\% in $100 \mathrm{~g}$ and comes from Akzo Nobel.

\section{Sample preparation}

\section{Dihydric alcohol PD preparation}

The dihydric alcohol PD contained acylhydrazone bonds and disulfide bonds are prepared according to the patent [25]. And the reaction is shown in Fig. 1.

\section{Polyurethane / polyamide amine (SH-WPU/PAMAM) gel preparation}

(1) Firstly, at room temperature, N2 atmosphere and condensate water, PEG-1000 (20 g) and DMSO (30 g) are added in the dry three-necked flask. Then, IPDI $(8.89 \mathrm{~g})$ is dropped in the system within $5 \mathrm{~min}$, and the DBTDL $(0.14 \mathrm{~g})$ is also added in the system after stirring for $15 \mathrm{~min}$. Then, the oil bath pan is heated to $75{ }^{\circ} \mathrm{C}$ and the system has been reacted for $3 \mathrm{~h}$. Finally, PEG-IPDI is obtained. The reaction is shown in Fig. 2, step 1.

(2) PD (17.84 g) dissolved by DMSO (18 g) is added in PEG-IPDI system. Then, the DBTDL $(0.09 \mathrm{~g})$ is also added in the system after stirring for $15 \mathrm{~min}$ and the system has been reacted at $75{ }^{\circ} \mathrm{C}$ for $5 \mathrm{~h}$. Finally, PEG-IPDI-PD is obtained. The reaction is shown in Fig. 2, step 2.

(3) HDI trimer ( $4.29 \mathrm{~g}$ ) is added in PEG-IPDI-PD system after its temperature falls at $25^{\circ} \mathrm{C}$. Then, the DBTDL $(0.05 \mathrm{~g})$ is added in the system after stirring for $15 \mathrm{~min}$. After that, the system is poured in the glass mold and reacts for $12 \mathrm{~h}$ at incubator $\left(75^{\circ} \mathrm{C}\right)$.Finally, polyurethane gels are obtained. The gels are then immersed in deionized water at room temperature and change water every $24 \mathrm{~h}$, and repeat three times until all the DMSO is replaced. Then, the moisture is removed in a vacuum drying oven $\left(100^{\circ} \mathrm{C}\right)$ and this step obtains self-healing polyurethane named SH-WPU. The reaction is shown in Fig. 2, step 3.

(4) The polyamide amine (PAMAM) is synthesized by N, N '-methylenebisacrylamide (MBA) and $\mathrm{N}$-aminoethylpiperazine (AEPZ). When the molar ratio of MBA to AEPZ $\mathrm{R}\left(\mathrm{R}=\frac{n_{M B A}}{n_{A E P Z}}\right)$ is greater than 1.2, PAMAM can form crosslinking structure [26]. In this paper, according previous studies on polyamide amine gels, $\mathrm{R}=1.25$ is used to prepare crosslinking polyamide amine. Firstly, MBA $(6.48 \mathrm{~g})$ and methanol $(30 \mathrm{~g})$ are mixed evenly under magnetic stirring machine in a $100 \mathrm{~mL}$ beaker. After that, AEPZ (4.32 g) is slowly added in the beaker. And stirring for $15 \mathrm{~min}$ makes the monomer solution mix evenly. Then, $30 \mathrm{~g}$ solution is poured in the glass mold quickly, and the SH-WPU (2.5 g) is soaked in the solution and the glass mold is sealed. After that, SH-WPU is swollen at room temperature completely and the solution which is not absorbed by SHWPU is removed by a dropper. Finally, they are put in the 
Fig. 1 The synthesis of PD

Step 1

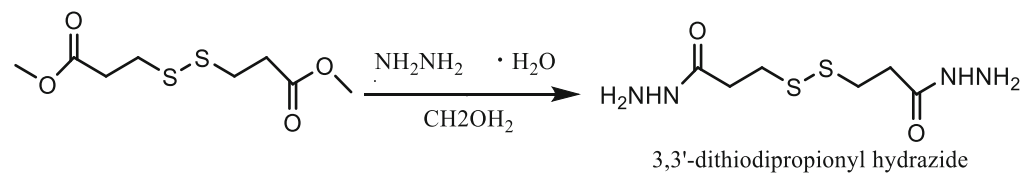

Step 2
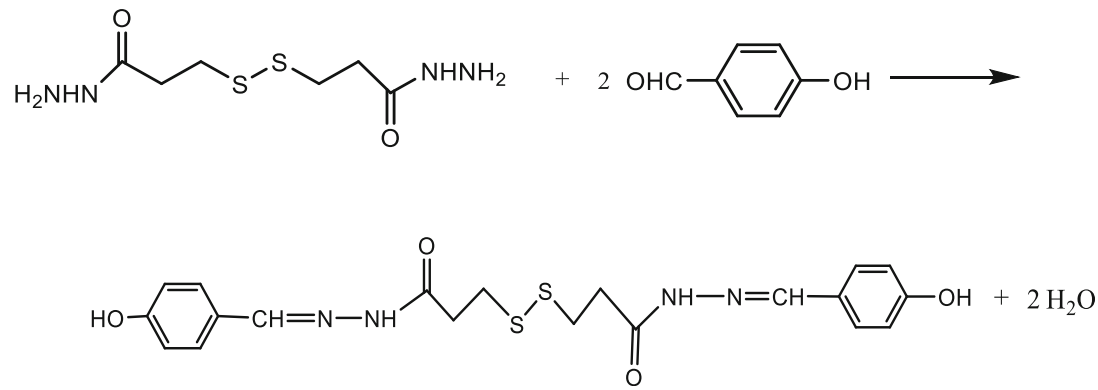

incubator $\left(50{ }^{\circ} \mathrm{C}\right)$ for $12 \mathrm{~h}$, and PAMAM can be crosslinked in the SH-WPU and IPN gel is obtained. The reaction is shown in Fig. 2, step 4.

\section{Polyurethane / polyamide amine (SH-WPU/PAMAM) hydrogel preparation}

The IPN gel which is swollen in 10 times deionized water. The water is changed every $24 \mathrm{~h}$ until all unreacted monomers are replaced, and the IPN hydrogel is prepared.

\section{Self-healing theory}

SH-WPU/PAMAM IPN hydrogel achieves self-healing by acylhydrazone bonds, disulfide bonds and hydrogen bonds.

The self-healing processes of reversible covalent bonds are shown in Fig. 3.

The self-healing processes of reversible non-covalent bonds are shown in Fig. 4.

Acylhydrazone bond is a kind of dynamic reversible covalent bonds obtained by the reaction of aldehyde groups and hydrazide groups and it can be broken and recombined by adjusting the $\mathrm{pH}$ value. Under the slightly acidic condition, the hydrogels can be healed by the reversible reaction of the acylhydrazone bonds, or under the neutral condition, the selfhealing can be achieved by the catalysis of the aniline. For disulfide bond, it can be self-healed under alkaline conditions, light or oxidation-reduction reaction. Under acidic conditions, the disulfide bonds are closed and the acylhydrazone bonds are opened, while the acylhydrazone bonds are closed and the disulfide bonds are opened under alkaline conditions [27, 28].

Polyamide amine is rich in hydrogen bonds which belong to reversible non-covalent bonds. Hydrogen bond is a kind of physical force, whinch is not as stable as covalent bond. But it also has a certain degree of reversibility and can achieve selfhealing.

\section{Tests and characterization}

\section{Characterization of infrared}

$\mathrm{KBr}$ pressing slice method is used for analysis of $\mathrm{PD}, \mathrm{SH}-$ WPU, By contrast, PEG-IPDI, PEG-IPDI-PD are coated on $\mathrm{KBr}$ wafers. Their infrared spectra are measured by VERTEX70 Fourier transform infrared-Raman spectroscopy (Bruker, Germany).

\section{Liquid absorption ratio studies}

The SH-WPU with the mass of $W_{S}$ is immersed in the PAMAM monomer solution with the mass concentration of $C w$ and the mass of $W p_{1}$. After swollen for $24 \mathrm{~h}$, the solution which is not absorbed by SH-WPU is removed with a dropper, and the removed solution is weighed (named $W p_{2}$ ), so the absorption rate (AR) of SH-WPU in PAMAM monomer solution is shown in Formula 1.

$A R=\frac{W_{P 3}}{W_{S}} \times 100 \%=\frac{W_{P 1}-W_{P 2}}{W_{S}} \times 100 \% \ldots \ldots \ldots \ldots$

Where, $W_{P 3}$ is the mass of PAMAM monomer solution absorbed by SH-WPU after $24 \mathrm{~h}$.

\section{Gels ratio studies}

In order to test the gels ratio of PAMAM in the SH-WPU, firstly, the IPN gels are immersed in 10 times deionized water, and we change water every $24 \mathrm{~h}$ and repeat three times until all 
Fig. 2 The preparation of selfhealing polyurethane / polyamide amine gel
Step 1

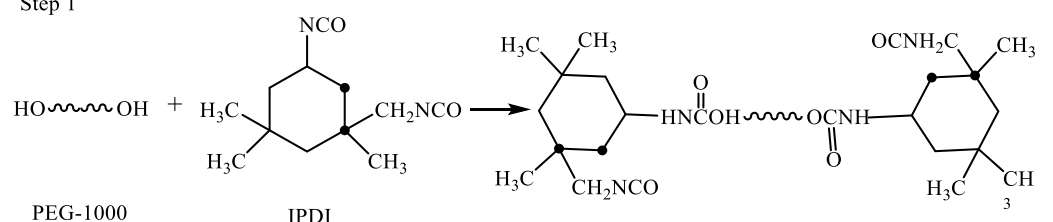

PEG-1000

IPDI

PEG-IPDI

Step 2

PEG-IPDI PD

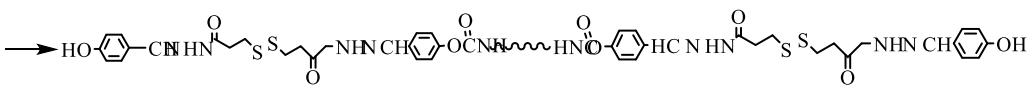
PEG-IPDI-PD

Step 3

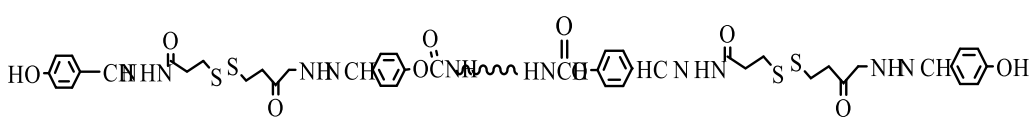
PEG-IPDI-PD

$+\left(\mathrm{O}=\mathrm{C}=\mathrm{N}-\mathrm{CH}_{2}\left(\mathrm{CH}_{2}\right)_{4} \mathrm{CH}_{2}-\mathrm{N}=\mathrm{C}=\mathrm{O}\right)_{3}$

$\longrightarrow$ SH-WPU

HDI trimer

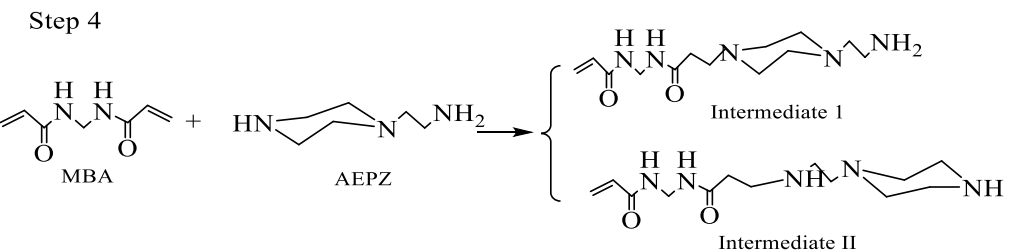

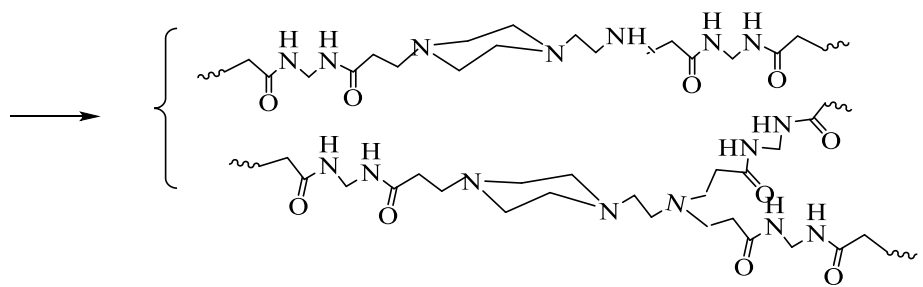

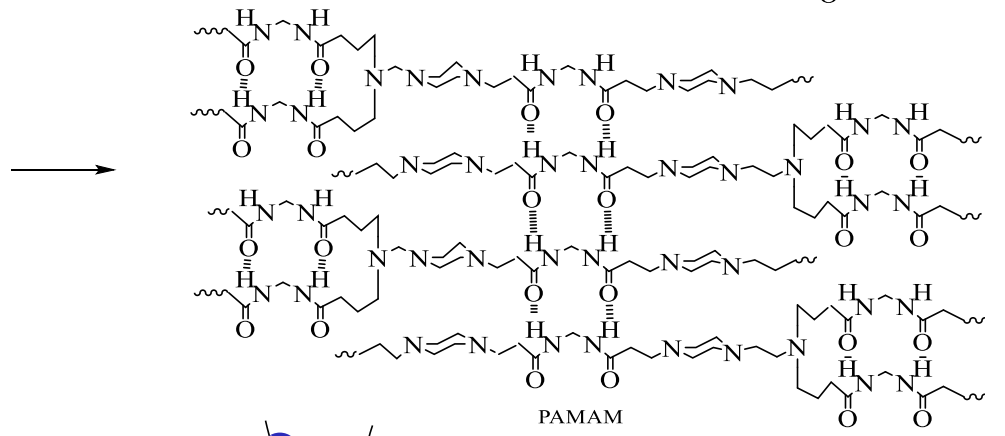

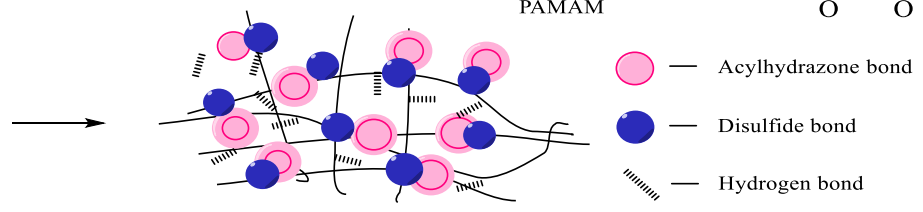


Fig. 3 Dynamic reversible processes of reversible covalent bonds

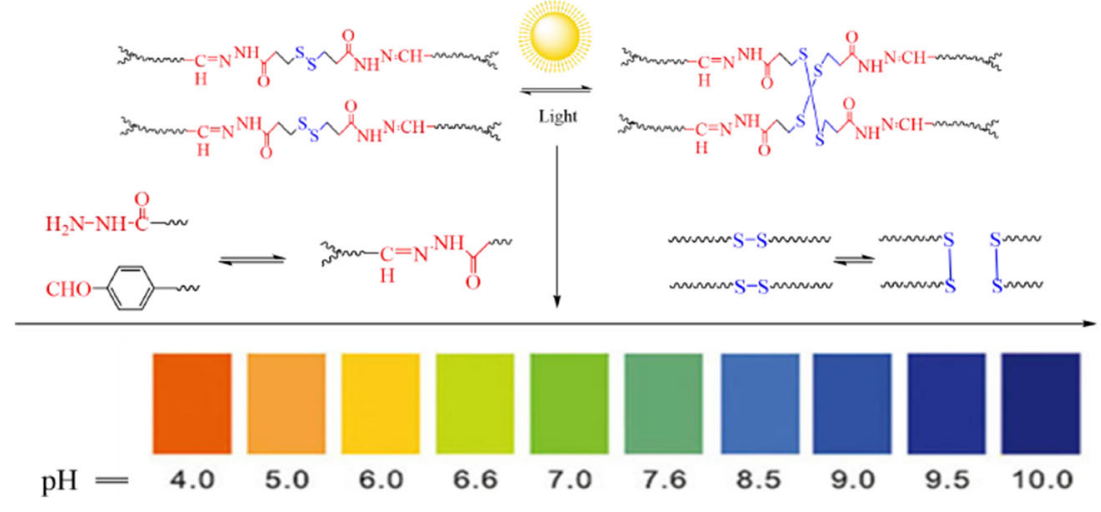

unreacted monomers are replaced. Then, the moisture is removed in a vacuum drying oven $\left(100{ }^{\circ} \mathrm{C}\right)$ until constant weight $W_{S P}$. The gels ratio (GR) is calculated by Formula 2 .

$\mathrm{GR}=\frac{W_{P}}{W_{P 4}} \times 100 \%=\frac{W_{S P}-W_{S}}{W_{P 3} \times C_{W}} \times 100 \%$

Where, $W_{P}$ is the mass of cross-linked PAMAM in constant weight $W_{S P}, W_{S}$ is the mass of SH-WPU, $W_{P 4}$ is the mass of PAMAM monomers absorbed by SH-WPU after $24 \mathrm{~h}, W_{p 3}$ is the mass of PAMAM monomers solution absorbed by SHWPU after $24 \mathrm{~h}, C_{W}$ is the mass concentration of PAMAM monomers solution.

\section{Equilibrium swelling ratio studies}

In order to test the equilibrium swelling ratio of the IPN hydrogels, firstly, the IPN gels are immersed in 10 times deionized water, and we change water every $24 \mathrm{~h}$ and repeat three times. Then, the moisture is removed in a vacuum drying oven $\left(100{ }^{\circ} \mathrm{C}\right)$ until constant weight $W_{S P}$. After that, the gels are immersed in deionized water again and we weigh the weight (named $W_{e}$ ) after up to swelling balance. The equilibrium swelling ratio (ESR) is calculated by Formula 3.

$\frac{\mathrm{ESR}=\boldsymbol{W e}-W s p}{W s p \times 100 \% \ldots \ldots \ldots \ldots \ldots}$

\section{Swelling kinetics studies}

The constant weight $W_{S P}$ hydrogel is immersed in deionized water and swollen at $37^{\circ} \mathrm{C}$. Then the hydrogel is taken at regular intervals and is wiped the surface water with filter papers and is weighed and recorded the weight. $t$ time hydrogel swelling ratio (SR) is calculated by Formula 4.

$\mathrm{SR}=\frac{W t-W d}{W d} \times 100 \%$

Where, $\mathrm{W} t$ is the hydrogel mass at $t$ time.

\section{Characterization of SEM}

In order to observe the compatibility of IPN hydrogels, the samples are placed in liquid nitrogen environment and have brittle fracture after $15 \mathrm{~min}$. Then, the JEM-1200 type scanning electron microscope (Japanese JEOL Corporation) is used to observe them.
Fig. 4 Dynamic reversible processes of reversible noncovalent bonds

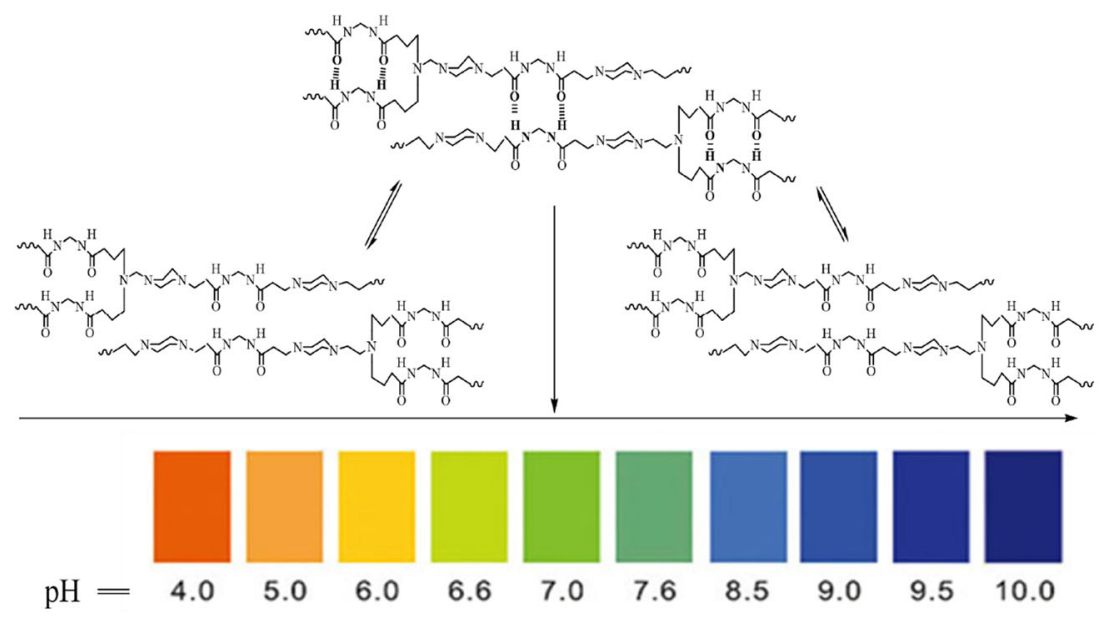




\section{Mechanical studies}

IPN hydrogel samples are prepared by rubber pneumatic slice machine (the size of samples are prepared according to GB/ T528-2009). The tensile properties of the samples are tested by Zwick/Roell/005 electronic universal testing machine (Zwick/Roell, Germany) and the tensile speed is $20 \mathrm{~mm} / \mathrm{min}$.

\section{Self-healing performance studies}

In order to quantify the self-healing efficiency, tensile tests are performed on virgin and healed IPN hydrogel samples. And these samples are tested by Zwick / Roell / 005 electronic universal testing machine (Zwick / Roell Company, Germany) and the tensile speed is $20 \mathrm{~mm} / \mathrm{min}$. The selfhealing efficiency is calculated by Formula 5 .

$\mathrm{R}(\sigma)=\frac{\sigma_{h}}{\sigma_{0}} \times 100 \% \ldots \ldots \ldots \ldots \ldots$

Where, $\sigma_{h}$ and $\sigma_{0}$ are the tensile strength of healed and virgin samples respectively.

\section{Results and discussion}

\section{IPN hydrogels preparation results}

From the Table 1, the mass ratio of SH-WPU to PAMAM is 100:60. The liquid absorption ratio of SH-WPU is higher, but the cross-linking of PAMAM is hindered by SH-WPU, leading to the cross-linking degree of PAMAM reducing and the gel ratio of PAMAM decreasing.

The preparation processes of IPN hydrogels are as follows.

From the Fig. 5, SH-WPU can be swollen completely in the PAMAM monomers solution and there is no swelling uneven phenomenon. And IPN gel is still transparent after PAMAM monomers crosslinked in SH-WPU. Besides, due to the poor sealing of the mold, a small amount of solvent is evaporated and leading to the volume of prepared IPN gels $\mathrm{C}$ is slight less than B. Thirdly, in the SH-WPU/PAMAM full-IPN hydrogels, SH-WPU is as water-borne polyurethane which owns hydrophilia. Also, PAMAM has amount of amide groups and amine groups which can absorb water, so the IPN hydrogel is prepared by IPN methanol gel swelled in the deionized water and replaced with deionized water.

\section{Analysis of IPN hydrogels molecular structure}

\section{Analysis of diatomic alcohol PD molecular structure}

Figure 6 is the infrared spectra of p-hydroxybenzaldehyde, $3,3^{\prime}$-dithiodipropionyl hydrazide and PD at 4000-500 $\mathrm{cm}^{-1}$ and $1700-1500 \mathrm{~cm}^{-1}$.

The stretching vibration absorption peak of $\mathrm{C}=\mathrm{O}$ bonds in the p-hydroxybenzaldehyde is at $1666 \mathrm{~cm}^{-1}$ and the absorption peak of $\mathrm{O}-\mathrm{H}$ bonds is at $3176 \mathrm{~cm}^{-1} .1454 \mathrm{~cm}^{-1}$, $1519 \mathrm{~cm}^{-1}$ and $1601 \mathrm{~cm}^{-1}$ are the absorption peaks of the benzene ring skeleton. In the FTIR spectrum of PD, $3182 \mathrm{~cm}^{-1}$ is $-\mathrm{OH}$ groups' stretching vibration absorption peak and $3095 \mathrm{~cm}^{-1}$ is the absorption peak of $\mathrm{C}-\mathrm{H}$ bonds on the benzene ring and $524 \mathrm{~cm}^{-1}$ is the absorption of -S-Sbonds. The acylhydrazone bonds are obtained by the reaction of aldehyde groups with hydrazine groups and the carbonyl aldehyde bonds disappear and acylhydrazone bonds are formed. The $-\mathrm{C}=\mathrm{O}$ groups absorption peak $1668 \mathrm{~cm}^{-1}$ of $\mathrm{p}$ hydroxybenzaldehyde and the $-\mathrm{NH}_{2}$ absorption peak $3297 \mathrm{~cm}^{-1}$ of 3,3'-dithiodipropionyl hydrazide both disappear, meanwhile, in the $\mathrm{PD}$ spectrum, the $-\mathrm{C}=\mathrm{N}$ groups stretching vibration absorption peak $1652 \mathrm{~cm}^{-1}$ appear, which indicates the formation of acylhydrazone bonds. So they can prove the reaction of p-hydroxybenzaldehyde with 3,3'dithiodipropionyl hydrazide and succeed in synthesis of patent's PD.
Table 1 IPN hydrogels preparation results

\begin{tabular}{lll}
\hline Sample name & Symbol & IPN Gel \\
\hline The Mass of SH-WPU & $W_{S} / \mathrm{g}$ & 2.50 \\
The Mass Concentration of PAMAM Monomer Solution & $C w / \%$ & 27 \\
The Mass of PAMAM Monomer Solution & $W p_{I} / \mathrm{g}$ & 30 \\
The Mass of the Solution Which is not Absorbed by the SH-WPU & $W p_{2} / \mathrm{g}$ & 18.95 \\
The Solution Mass Absorbed by SH-WPU after 24 Hours & $W_{P 3} / \mathrm{g}$ & 11.05 \\
The Mass of PAMAM Monomers Absorbed by SH-WPU after 24 Hours & $W_{P 4} / \mathrm{g}$ & 2.98 \\
The Liquid Absorption Ratio of SH-WPU & $A R / \%$ & 442.0 \\
The Mass of IPN Polymer Which is Removed Solvent and Unreacted Monomers & $W_{S P} / \mathrm{g}$ & 3.99 \\
The Mass of Cross-linked PAMAM & $W_{P} / \mathrm{g}$ & 1.49 \\
The Gels Ratio of PAMAM in SH-WPU & $G R / \%$ & 50.00 \\
The Mass Ratio of SH-WPU to PAMAM & & $100: 60$ \\
\hline
\end{tabular}



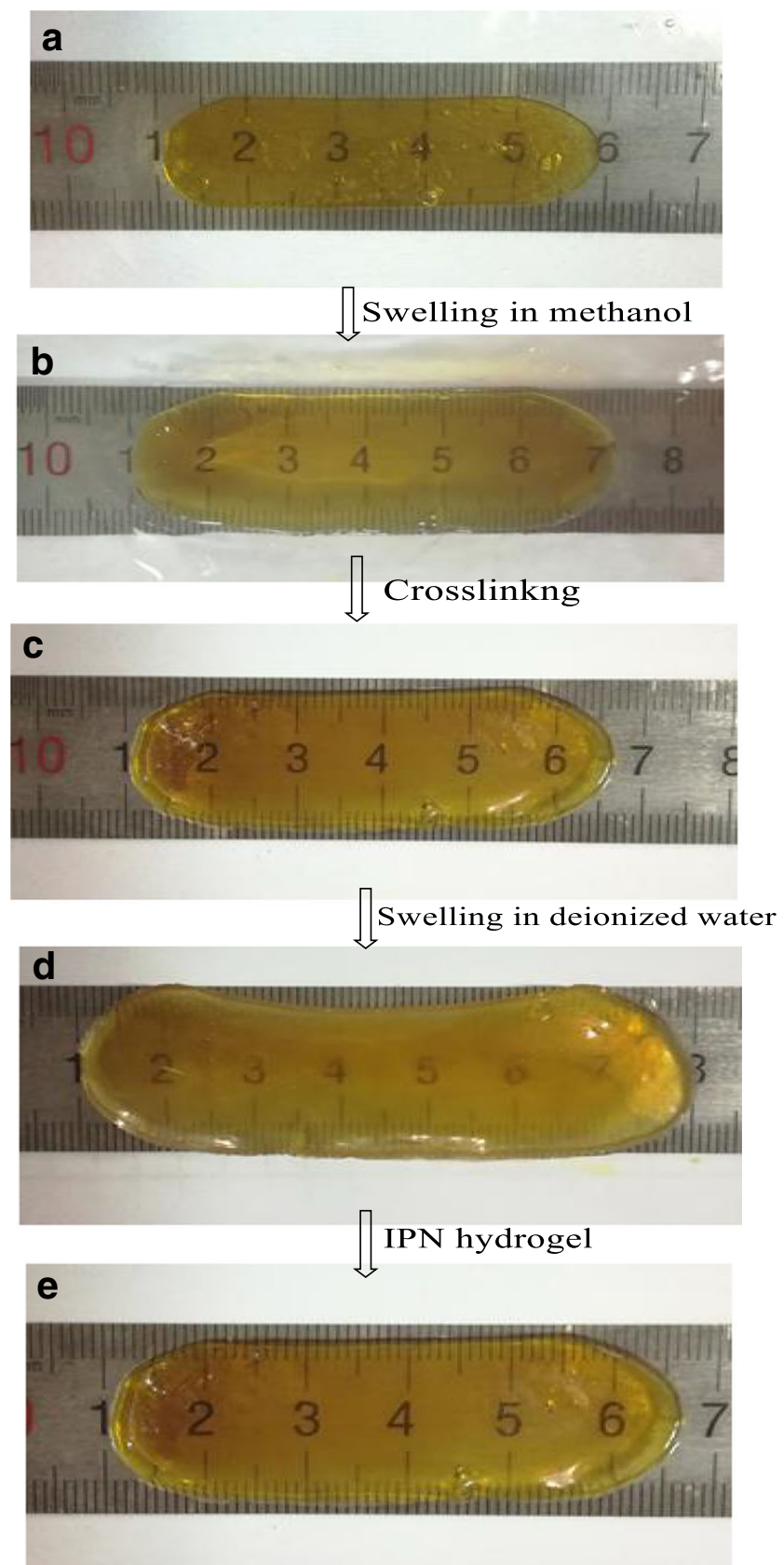

Fig. 5 IPN hydrogels preparation processes. Where, a SH-WPU is immersed in the methanol solution, b SH-WPU is swollen completely in the methanol solution and $\mathbf{c}$ IPN gel is obtained by crosslinking, d IPN methanol gel is swollen in deionized water, e IPN hydrogel is obtained

\section{Analysis of SH-WPU molecular structure}

On the PEG-1000 spectrum, $3421 \mathrm{~cm}^{-1}$ is hydroxyl stretching vibration absorption peak. On the IPDI spectrum, $2262 \mathrm{~cm}^{-1}$ is - $\mathrm{NCO}$ groups stretching vibration absorption peak. By contrast, on the PEG-IPDI spectrum, the -NCO groups still exist, and it illustrate that the system still contains isocyanate groups after PEG-1000 and IPDI reaction completely. Besides, $1717 \mathrm{~cm}^{-1}$ is the infra-red absorption peak of the amide I band
$(-\mathrm{C}=\mathrm{O})$, and $1547 \mathrm{~cm}^{-1}$ is the infra-red absorption peak of the amide II band (N-H bending and $\mathrm{C}-\mathrm{N}$ stretching vibration), and $1250 \mathrm{~cm}^{-1}$ is the infra-red absorption peak of the amide III band $(\mathrm{C}-\mathrm{N}-\mathrm{H})$, and $3568 \mathrm{~cm}^{-1} \sim 3217 \mathrm{~cm}^{-1}$ is the $-\mathrm{NH}$ stretching vibration peak region, and $3094 \mathrm{~cm}^{-1}$ is $1547 \mathrm{~cm}^{-1}$ frequency doubling peak. Above analyses illustrate that PEG-1000 and IPDI could react with each other and produce carbamate groups (-CONH-).

On the PD spectrum, $3172 \mathrm{~cm}^{-1}$ is hydroxyl stretching vibration absorption peak. On the PEG-IPDI spectrum, $2262 \mathrm{~cm}^{-1}$ is the characteristic absorption peak of isocyanate groups. By contrast, on the PEG-IPDI-PD spectrum, the isocyanate infra-red absorption peak disappears, which illustrates the isocyanate group reaction completely. Besides, $3533 \mathrm{~cm}^{-1} \sim 3259 \mathrm{~cm}^{-1}$ region is the combined absorption peaks of -NH groups and hydroxyl groups.

On the HDI trimer spectrum, $2274 \mathrm{~cm}^{-1}$ is the characteristic absorption peak of isocyanate groups. By contrast, - $\mathrm{NCO}$ groups disappear on SH-WPU spectrum, meanwhile, the broad -OH absorption peak in the PEG-IPDI-PD spectrum disappears and forms a narrower - $\mathrm{NH}$ absorption peak $\left(3304 \mathrm{~cm}^{-1}\right)$ in the SH-WPU spectrum. Above analyses illustrat that PEG-IPDI-PD and HDI trimer can react completely (Fig. 8).

\section{Analysis of SH-WPU/PAMAM IPN hydrogel molecular structure}

On the SH-WPU spectrum, $1717 \mathrm{~cm}^{-1}, 1547 \mathrm{~cm}^{-1}$ and $1250 \mathrm{~cm}^{-1}$ is the infra-red absorption peak of the amide I band, amide II band and amide III band respectively. $3568 \mathrm{~cm}^{-1} \sim 3217 \mathrm{~cm}^{-1}$ is the $-\mathrm{NH}$ stretching vibration peak region. Above infra-red absorption peaks are characteristic absorption peaks of carbamate (-CONH-). On the PAMAM spectrum, $1613 \mathrm{~cm}^{-1}$ is $-\mathrm{C}=\mathrm{O}$ stretching vibration absorption peak, which moves toward a low peak due to hydrogen bonding and illustrates that the system contains lots of reversible non-covalent bonds (hydrogen bonds). $1505 \mathrm{~cm}^{-1}$ is the absorption peak of the amide II band, and $3246 \mathrm{~cm}^{-1}$ is -NH stretching vibration absorption peak. By contrast, the infrared spectrum of the IPN gel coincides with the sum of the infrared spectrum of the SH-WPU and PAMAM, which illustrates that in the preparation of IPN gels, no complex chemical bonds are formed, or both of them are not copolymerization. Besides, on the PAMAM spectrum and SH-WPU spectrum, $2354 \mathrm{~cm}^{-1}$ is $\mathrm{CO}_{2}$ absorption peak, which has no effect on the test results (Fig. $8)$.

\section{IPN hydrogels equilibrium swelling ratio}

In the Table 2, the equilibrium swelling ratio (ESR) of IPN hydrogel is $354 \%$. The PAMAM molecular skeleton is rich in 

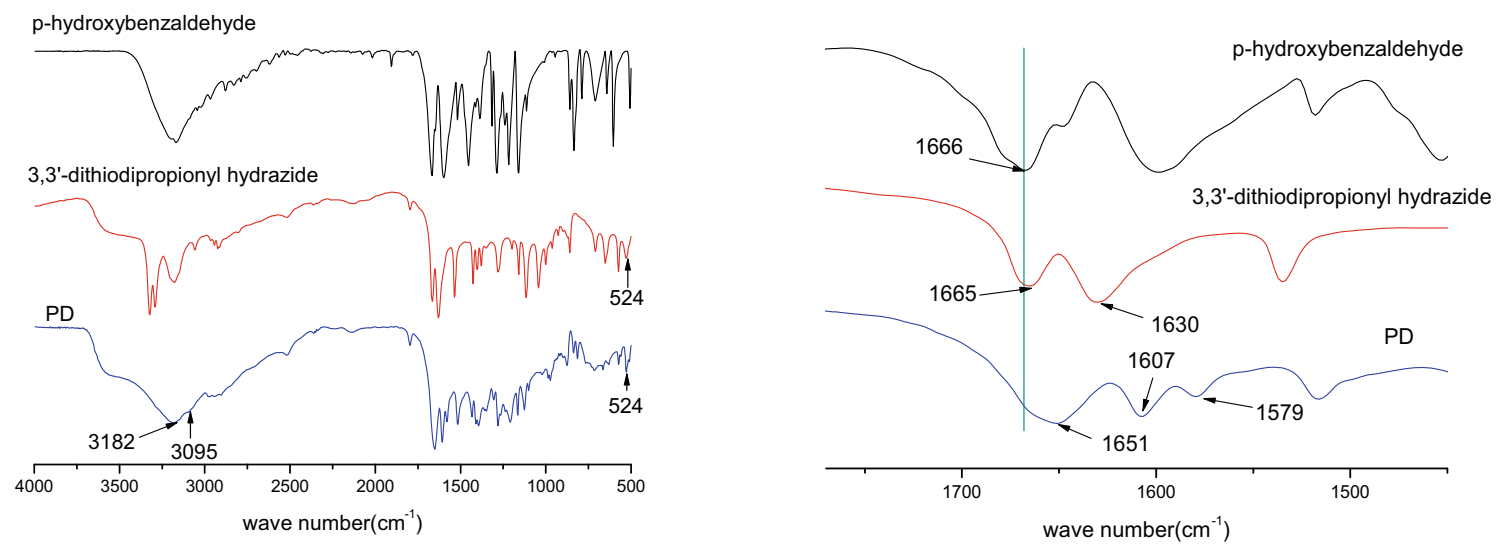

Fig. 6 P-hydroxybenzaldehyde, 3,3'-dithiodipropionyl hydrazide and PD FTIR spectra

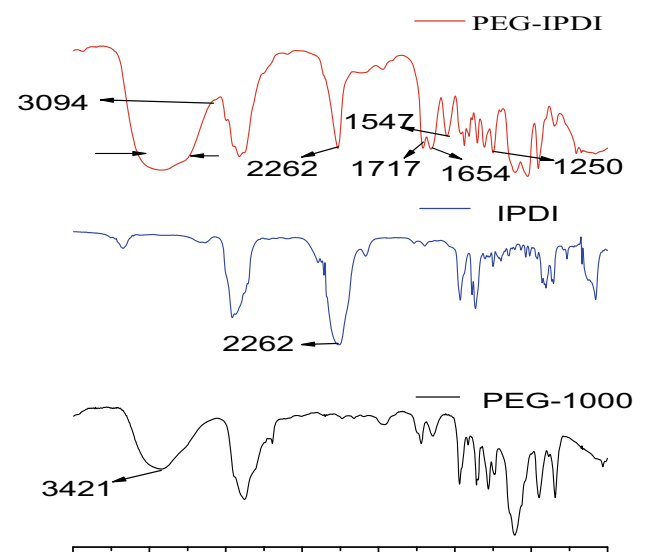

4000350030002500200015001000500 Wave number $\left(\mathrm{cm}^{-1}\right)$
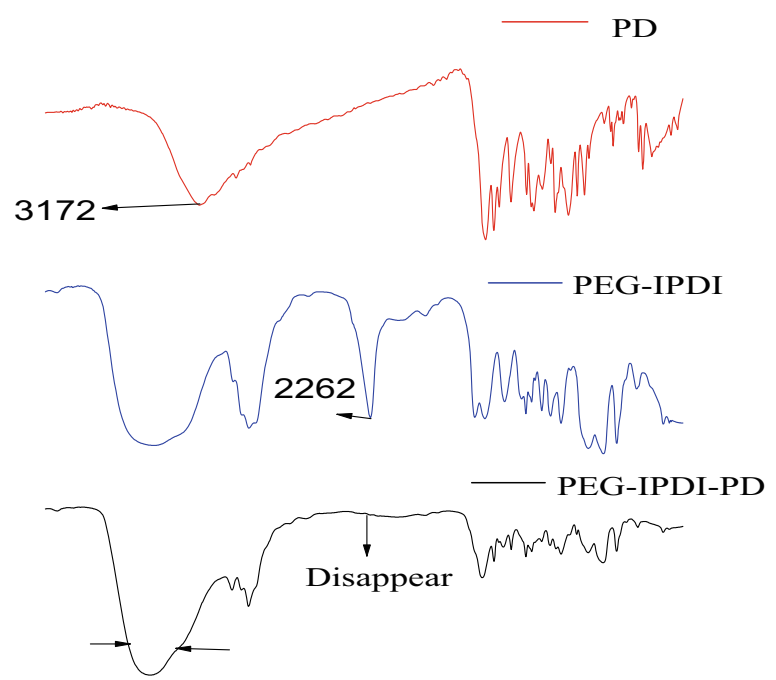

4000350030002500200015001000500 Wave number $\left(\mathrm{cm}^{-1}\right)$
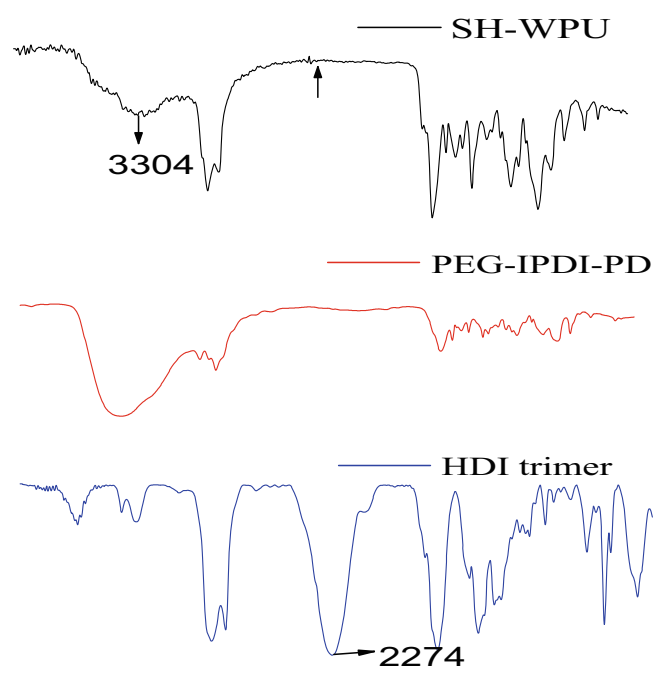

\section{0}

Wave number $\left(\mathrm{cm}^{-1}\right)$

Fig. 7 PEG-IPDI, IPDI and PEG-1000; PD, PEG-IPDI and PEG-IPDI-PD; SH-WPU, PEG-IPDI-PD and HDI trimer FTIR spectra 


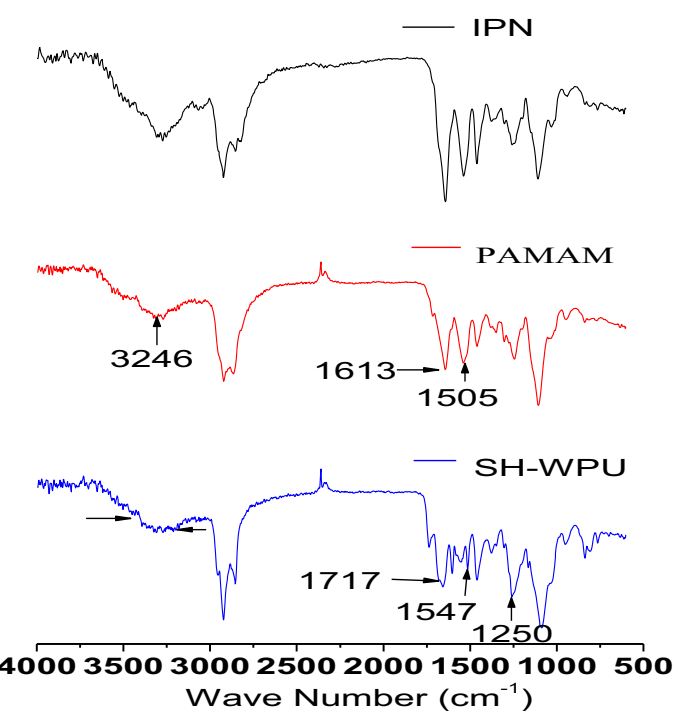

Fig. 8 IPN, PAMAM and SH-WPU FTIR spectra

amide groups and amine groups which are beneficial to develop high density hydrogen network. Meanwhile, PEG-1000 and IPDI also have hydrophilia. So the IPN hydrogel owns high hydrophilia and makes the equilibrium swelling ratio high.

\section{Swelling kinetics of IPN hydrogels}

As is shown in Fig. 9, IPN hydrogels contain amount of hydrophilic groups, the solvent diffuses into the hydrogel network at the beginning of the swelling, causing the volume of hydrogel to expand. And the polymer chains are relaxed under solvation and absorb a lot of water, so the swelling rate is high. Also, in the later stage of swelling, the groups which absorb water are reduced, and the polymer chains extend to the three-dimensional space, and the hydrogel network is retracted by the stress, so the swelling rate decreases and achieves swelling balance.

\section{Study on the compatibility of IPN hydrogels}

\section{SEM}

Figure 10 is the scanning electron micrograph of the brittle section magnified 5000 times and 10,000 times, respectively. It can be seen from the figure that the material is a onephase structure, indicating that polyurethane and polyamide have good compatibility and no phase separation occurs.

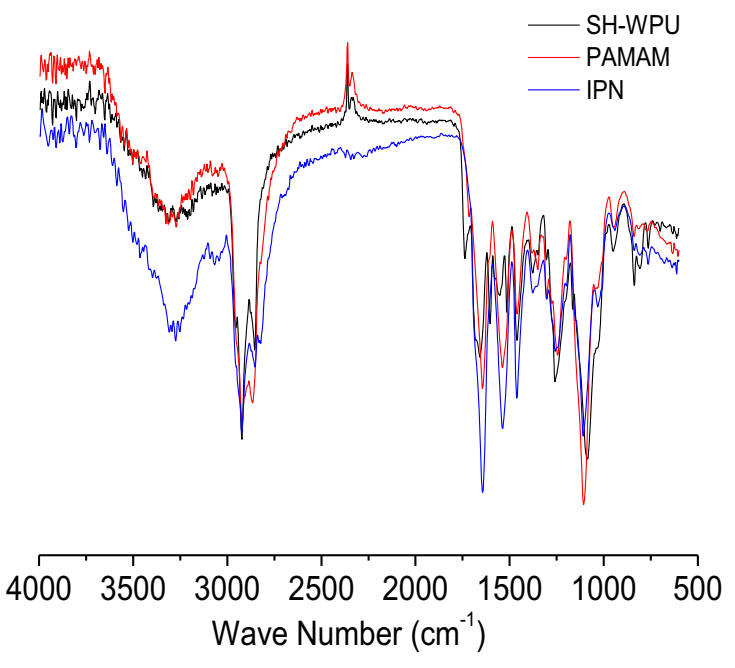

Because polyurethane and polyamide are physically blended by chemical methods, forming IPN structure, which is better than general physical blending compatibility. In addition, hydrogen bond can also be formed between polyurethane and polyamide, which is also beneficial to the compatibility of materials.

\section{IPN hydrogels tensile properties}

In Fig. 11, with the increase of containing water in hydrogels, the tensile strength is decreasing and the elongation at break is also increasing, while the water percentage is $45 \%$ and $60 \%$, the tensile strength and the elongation at break are both decreasing. In addition, the hydrogel can be measured up to $60 \%$ water content, which tensile strength is $0.0899 \mathrm{MPa}$, elongation at break is $39.95 \%$. If water content increases again, then they will be broken by fixture and cannot be measured. So we choose the hydrogel which water percentage is $30 \%$ to research its self-healing properties.

\section{Self-healing performance characterization}

\section{Self-healing performance qualitative characterization}

The self-healing diagrams under light conditions are shown in Fig. 12A, where light uses fluorescent lamp, voltage $220 \mathrm{~V}$, power $9 \mathrm{~W}$, U-shaped lamp (Bao Shida Company).

Table 2 Equilibrium swelling ratio of IPN hydrogels

\begin{tabular}{lllll}
\hline Name & SH-WPU and PAMAM Mass Ratio & constant weight & Equilibrium Swelling Weight & Equilibrium Swelling Ratio \\
\hline Symbol & - & $W_{S P} / \mathrm{g}$ & $W_{e} / \mathrm{g}$ & $E S R / \%$ \\
IPN hydrogel & $100: 60$ & 1.00 & 4.54 & 354 \\
\hline
\end{tabular}




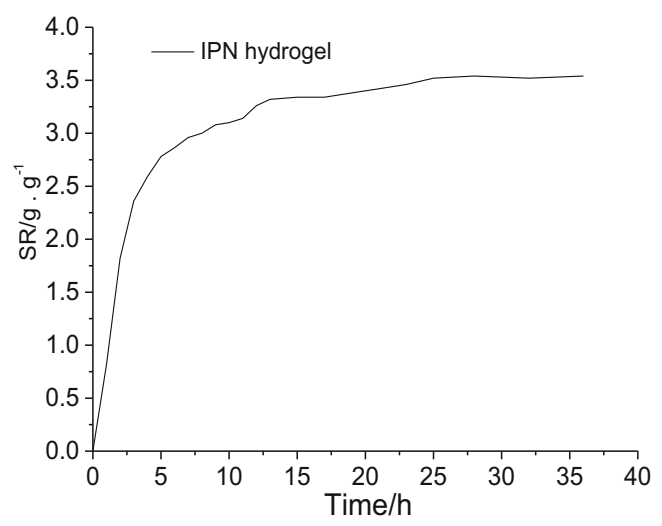

Fig. 9 Swelling curve of IPN hydrogel

Figure 12A shows the self-healing processes of IPN hydrogel under light environment. The virgin sample can be stretched from $4.8 \mathrm{~cm}$ to $9.0 \mathrm{~cm}$, after light for $48 \mathrm{~h}$, the selfhealing sample can withstand the weight of $20 \mathrm{~g}$ and can be stretched to $8.2 \mathrm{~cm}$, indicating that IPN hydrogel owns selfhealing ability. Because PAMAM contains a lot of hydrogen bonds, the fresh section is put together and the hydrogel can be recovered in a short time. Besides, SH-WPU molecular chains contain disulfide bonds which can achieve reversible dynamic balance in the light conditions, and achieve self-healing.

Figure 12B shows the self-healing processes of IPN hydrogel under acid environment. The cut is put together and adds $0.1 \mathrm{~mL}$ glacial acetic acid. After $48 \mathrm{~h}$, the cut can be connected together. When the sample is put vertically as E, the self-healing place can withstand its own gravity, but it cannot withstand the weight of $20 \mathrm{~g}$ and cannot be stretched after self-healing, so we know that its self-healing efficiency is lower under acid environment. In the acid environment, only acylhydrazone bonds can achieve dynamic reversible, while the disulfide bonds and hydrogen bonds are inhibited, so the self-healing effect is poor.
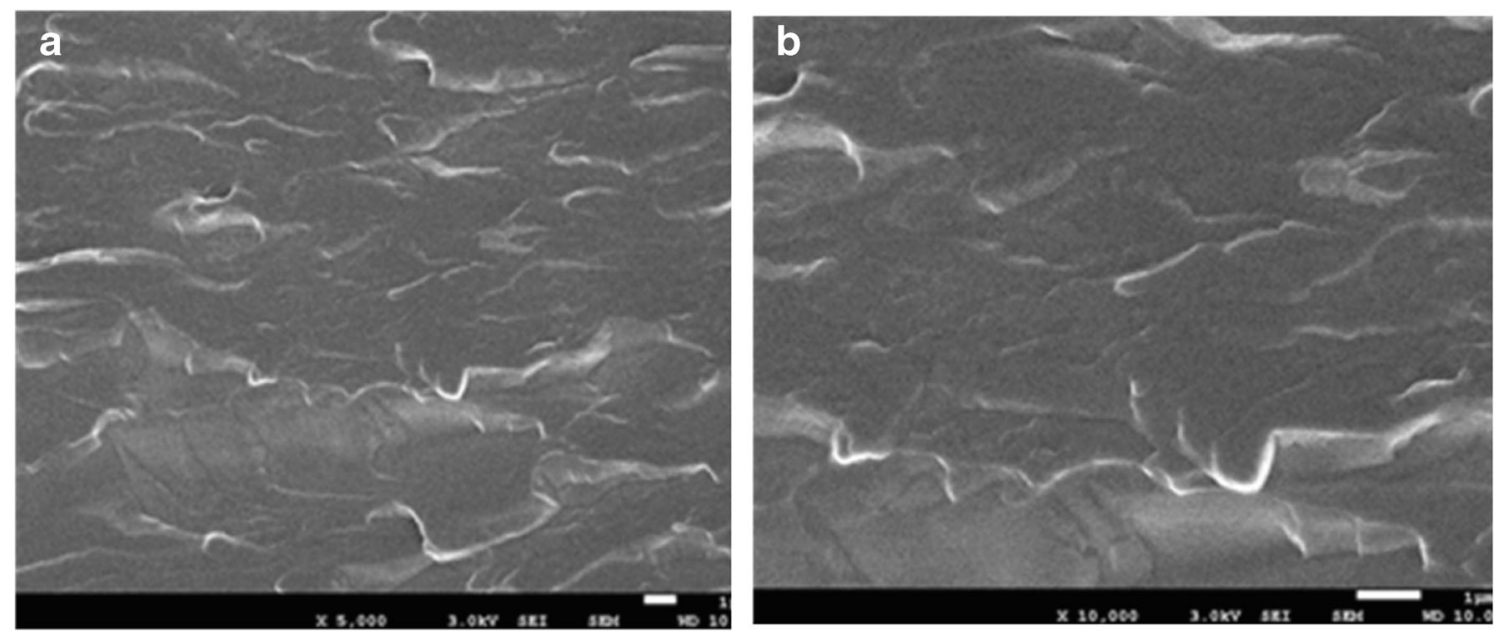

Fig. 10 SEM images of IPN hydrogel, Where, a was enlarged 5000 times and $\mathbf{b}$ was enlarged 10,000 times

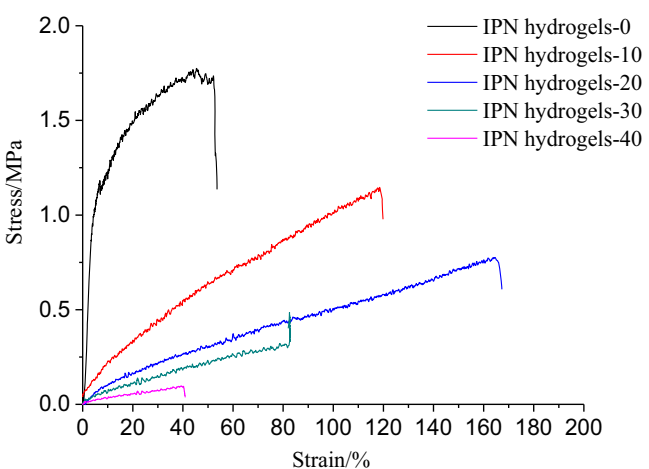

Fig. 11 IPN hydrogens-0 represents a hydrogel with a water content of 0 , IPN hydrogen-10 represents a hydrogels with a water content of $10 \%$, IPN hydrogens-20 represents a hydrogel with a water content of $20 \%$, IPN hydrogens-30 represents a hydrogel with a water content of $30 \%$, IPN hydrogens- 40 represents a hydrogel with a water content of $40 \%$

Figure $12 \mathrm{C}$ shows the self-healing processes of IPN hydrogel under alkaline environment. The cut is put together and adds $0.1 \mathrm{~mL}$ triethylamine. After $48 \mathrm{~h}$, the cut can be connected together. When the sample is put vertically as E, the self-healing place can withstand $20 \mathrm{~g}$, also it can be stretched to $5.6 \mathrm{~cm}$ after self-healing, meanwhile, it appears crack. So we know that its selfhealing efficiency is lower under alkaline conditions. In the alkaline environment, only disulfide bonds can achieve dynamic reversible, while the acylhydrazone bonds and hydrogen bonding can be inhibited, so that the self-healing effect is poor. However, since the disulfide bond energy is stronger than the acylhydrazone bond's, the sample can be stretched after self-healing in the alkaline conditions.

In conclusion, the hydrogel can obtain the highest selfhealing efficiency under the light conditions, while the self-healing effect is not ideal in acidic or alkaline conditions. So we research its self-healing efficiency under light conditions. 


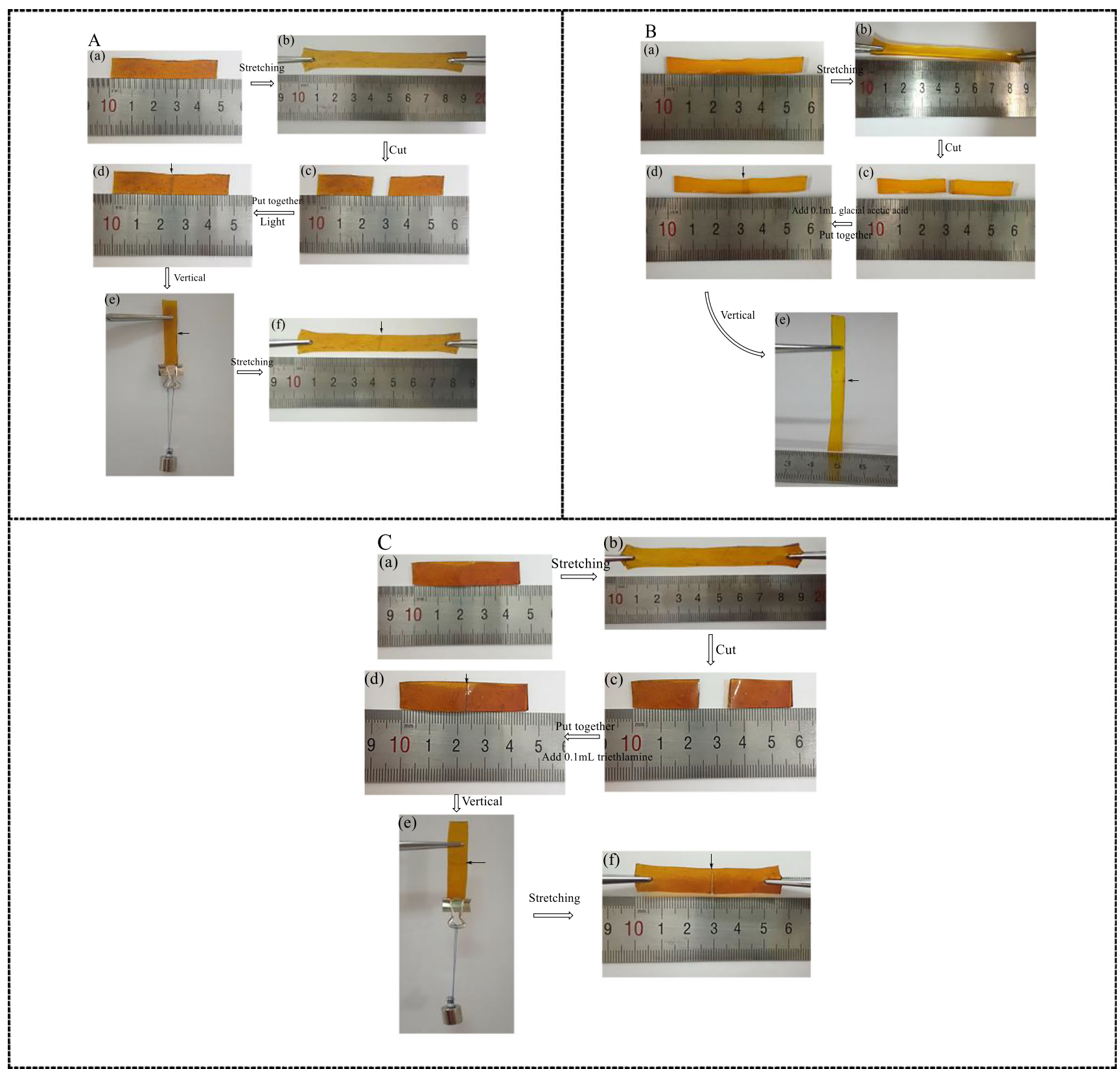

Fig. 12 A Light self - healing processes of IPN hydrogel (Where, (a) is the virgin sample $4.8 \mathrm{~cm}$, (b) is stretched to $9.0 \mathrm{~cm}$ of the virgin sample, (c) is cut, (d) is put together, (e) is placed vertically after self-healing, (f) is stretched to $8.2 \mathrm{~cm}$ of the self-healing sample, " $\rightarrow$ "shows the self-healing placement), B Acid self - healing processes of IPN hydrogel (Where, (a)0 is the virgin sample $5.5 \mathrm{~cm}$, (b) is stretched to $8.6 \mathrm{~cm}$ of the virgin sample, (c) is cut, (d) is put together, (e) is placed vertically after self-healing, " $\rightarrow$ "shows the self-healing placement) $\mathbf{C}$ Alkaline self - healing processes of IPN hydrogel (Where, (a) is the virgin sample $4.6 \mathrm{~cm}$, (b) is stretched to $9.0 \mathrm{~cm}$ of the virgin sample, (c) is cut, (d) is put together, (e) is placed vertically after self-healing, (f) is stretched to $5.6 \mathrm{~cm}$ of the self-healing sample, " $\rightarrow$ "shows the self-healing placement)

\section{Self-healing performance quantitative characterization}

Figure 13 shows IPN hydrogel self-healing tensile properties at $1 \mathrm{~h}, 24 \mathrm{~h}$ and $48 \mathrm{~h}$ respectively. Combined with Table 3, we know that when it is $1 \mathrm{~h}, 24 \mathrm{~h}$ and $48 \mathrm{~h}$, the self-healing ratio of IPN hydrogel is $31.58 \%, 49.84 \%$ and $87.35 \%$ respectively. Lighting for $1 \mathrm{~h}$, hydrogen bonds which belongs to the reversible non-covalent bonds and requires less energy and shorter time to repair. Although the system contains disulfide bonds, which belongs to reversible covalent bonds and requires more energy and longer time to repair, so under light $1 \mathrm{~h}$, the self-healing efficiency is up to the virgin tensile strength of one third. Lighting for $24 \mathrm{~h}$, both hydrogen bonds and disulfide bonds establish reversible dynamic equilibrium to make the hydrogels achieve self-healing. Self-healing for $48 \mathrm{~h}$, the 


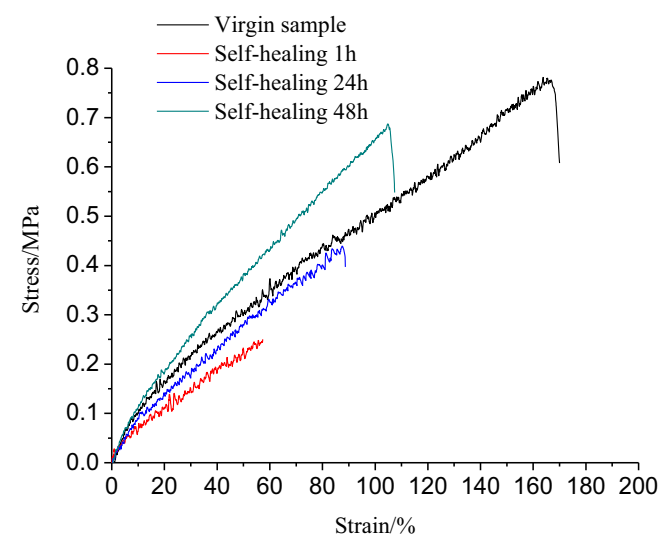

Fig. 13 Tensile properties of IPN hydrogel with different self-healing time

self-healing ratio of IPN hydrogel is up to $87.35 \%$. And broken disulfide bonds all establish dynamic reversible equilibrium, which interacts that the hydrogen bonds make the material heal. Besides, the damaged materials do not guarantee $100 \%$ to break self-healing bonds, so the selfhealing ratio cannot guarantee $100 \%$. At the same time, during the light conditions, the hydrogel's water is partially volatilized, so the slope of the curves increase and the modulus of the materials also increase.

\section{Conclusions}

In this paper, we synthesize self-healing IPN hydrogels which contain reversible covalent bonds and reversible non-covalent bonds. The IPN hydrogels are produced by waterborne polyurethane (SH-WPU) and cross-linked polyamide amine (PAMAM), where SH-WPU is the first networking and contains reversible covalent bonds, and PAMAM is the second networking and contains reversible non-covalent bonds. In this paper, disulfide bonds and acylhydrazone bonds can be combined in SH-WPU and hydrogen bonds existed in PAMAM. Also, FTIR tests show that IPN structure can be produced by SH-WPU and PAMAM. SEM test results illustrate that the compatible of SH-WPU and PAMAM is good. Self-healing for $1 \mathrm{~h}$, $24 \mathrm{~h}$ and $48 \mathrm{~h}$, the self-healing efficiency is $31.58 \%$, $49.84 \%$ and $87.35 \%$ respectively. The hydrogels can be self-healing under acid, alkaline or light conditions and at room temperature. This effect achieved the desired effect

Table 3 Self-healing efficiency of IPN hydrogel with different time

\begin{tabular}{lllll}
\hline & Virgin & Light 1 h & Light 24 h & Light 48 h \\
\hline Tensile Strength/MPa & 0.7739 & 0.2444 & 0.3857 & 0.6760 \\
Self-healing Ratio/\% & - & $31.58 \%$ & $49.84 \%$ & $87.35 \%$ \\
\hline
\end{tabular}

and the repair effect is more obvious than previous research results.

Supplementary Information The online version contains supplementary material available at https://doi.org/10.1007/s10965-020-02155-9.

Funding information This work was supported by the National Natural Science Foundation of China (grant no. 51103078).

\section{Compliance with ethical standards}

Conflict of interest The authors declare that they have no conflict of interest.

Open Access This article is licensed under a Creative Commons Attribution 4.0 International License, which permits use, sharing, adaptation, distribution and reproduction in any medium or format, as long as you give appropriate credit to the original author(s) and the source, provide a link to the Creative Commons licence, and indicate if changes were made. The images or other third party material in this article are included in the article's Creative Commons licence, unless indicated otherwise in a credit line to the material. If material is not included in the article's Creative Commons licence and your intended use is not permitted by statutory regulation or exceeds the permitted use, you will need to obtain permission directly from the copyright holder. To view a copy of this licence, visit http://creativecommons.org/licenses/by/4.0/.

\section{References}

1. Roy N, Buhler E, Lehn JM (2014) Double dynamic self-healing polymers: supramolecular and covalent dynamic polymers based on the bis-iminocarbohydrazide motif[J]. Polym Int 63(8):14001405

2. Cassagneau T, Guérin F, Fendler JH (2000) Preparation and characterization of ultrathin films layer-by-layer self-assembled from graphite oxide nanoplatelets and polymers[J]. Langmuir 16(18): 7318-7324

3. Kessler MR, White SR (2001) Self-activated healing of delamination damage in woven composites[J]. Compoos Part Appl S 32(5): 683-699

4. Brown EN, Sottos NR, White SR (2003) In situ poly(urea-formaldehyde) microencapsulation of dicyclopentadiene[J]. J Microencapsul 20(6):719-730

5. Brown EN, White SR, Sottos NR (2005) Retardation and repair of fatigue cracks in a microcapsule toughened epoxy composite[J]. Compos Sci Technol 65(15-16):2474-2480

6. Dry C (1992) Smart building materials which prevent damage or repair themselves[J]. MRS Proceedings 276:311-314

7. Kersey FR, Loveless DM, Craig SL (2007) A hybrid polymer gel with controlled rates of cross-link rupture and self-repair. J R Soc Interface 4(13):373-380

8. Liu YL, Hsieh CYJ (2006). Polym Sci Part A Polym Chem 44:905913

9. Yu Z, Feng L, Chai C (2016) Structure and repair behavior of self repairing polyurethane based on Diels - Alder reaction[J]. J Polym Sci 11:1579-1586

10. Tang C, Deng G, Li F, Jiang H, Chen Y (2010) Covalent crosslinked polymer gels with reversible sol-gel transition and selfhealing properties[J]. Macromoleculers 43(3):1191-1194 
11. Deng GH, Li FY, Yu HX (2012) Dynamic hydrogels with an environmental asaptive self-healing ability and dual responsive solgel transitions[J]. ACS Macro Lett 1(2):275-279

12. Gong Z, Zhang G, Sun R, Wang Z (2015) Intrinsic self - repairing polymer materials based on non - covalent bond and their applications[J]. Polym Bull 4:1-11

13. Cordier P, Tournilhac F, Ziakovic CS, Leibler L (2008). Nature [J] 451:977-980

14. Zhang MM, Xu DH, Yan XZ (2012) Self-healing supramolecular gels formed by erown ether based host-guest interactions[J]. Angew Chem Int Ed 51(28):7011-7015

15. Tuncaboylu DC, Sari M, Oppermann W (2011) Tough and selfhealing hydrogels formed via hydrophobic[J]. Macromolecules 44: 4997-5005

16. Chen Y, Dong K, Liu Z (2012) High strength double network polymer hydrogels: performance, progress and prospect[J]. Sci China Technol Sci 42(8):859-873

17. Li J, Llleperuma WRK, Suo Z (2014) Hybrid hydrogels with extremely high stiffness and toughness[J]. ACS Macro Lett 3(6):520 523

18. Peak CW, Wilker JJ, Schmidt G (2013) A review on tough and sticky hydrogels[J]. Colloid Polym Sci 291(9):2031-2047

19. Illescas J, Sanna R, Alzari V (2013) Organic-inorganic interpenetrating polymer networks and hybrid polymer materials prepared by frontal polymerization[J]. J Polym Sci A Polym Chem 51(21): 4618-4625

20. Cui L, Jia J, Xiong Z (2014) Preparation and properties of carboxymethyl chitosan / sodium alginate semi - interpenetrating network hydrogels[J]. J Polym Sci 3:361-368
21. Tang Q, Sun H, Ao H (2008) Preparation of hydrogel with high compressive strength acrylate / polysiloxane interpenetrating network[J]. Funct Mater 38(12):1993-1996

22. Djonlagic J, Petrovic ZS (2004) Semi-interpenetrating polymer networks composed of poly(N-isopropylacrylamide) and polyacrylamide hydrogels[J]. J Polym Sci B Polym Phys 42(21):3987-3999

23. Nakajima T, Sato H, Zhao Y (2012) A universal molecular stent method to toughen any hydrogels based on double network concept[J]. Adv Funct Mater 22(21):4426-4432

24. Suekama TC, Hu J, Kurokawa T (2013) Double-network strategy improves fracture properties of chondroitin sulfate networks[J]. ACS Macro Lett 2(2):137-140

25. Wei Y, Bai Y Dihydric alcohols containing acylhydrazone bonds, dihydric alcohols containing acylhydrazone bonds and disulfide bonds, self-repairing polyurethane elastomers and methods for their preparation: China 201610473397.5[P].2016-11-16

26. Zhang Y (2008) Synthesis and functionalization of hyperbranched polyamide amines[D]. Shanghai Jiaotong University, Shanghai

27. Orrillo AG, Escalante AM, Furlan RLE (2008) Covalent double level dynamic combinatorial libraries: selectively addressable exchange processes[J]. Chem Commun 42:5298-5300

28. Rodriguez-Docampo Z, Otto S (2008) Orthogonal or simultaneous use of disulfide and hydrazine exchange in dynamic covalent chemistry in aqueous solution[J]. Chem Commun 42:5301-5303

Publisher's note Springer Nature remains neutral with regard to jurisdictional claims in published maps and institutional affiliations. 